\title{
Design and Implementation of Mobile Intelligent Service System for City Sports Venues
}

\author{
HAN Fang ${ }^{1, a}$, BO Xuesong ${ }^{2}$ \\ ${ }^{1,2}$ Physical education department of hebei normal university, Shijiazhuang 050024,China \\ ahanfanghb@126.com
}

Keywords: City Sports Venues; Mobile Devices; Intelligent Service; Internet of Things (IoT).

\begin{abstract}
Internet information service system integrating the infrastructure layer access to huge amounts of data which is geared to the needs of all kinds of Internet of things applications provide tracking and monitoring and control and the key of the information service platform. In this paper, we conduct research on the design and implementation of mobile intelligent service system for city sports venues. Internet of things through a variety of sensing equipment to obtain data has massive, heterogeneous, high dimension, redundancy, time series and space dispersion, etc. The proposed system helps to modify the current situation of sport service and we plan to use the current platform to more related infrastructures. Moreover, we will polish the structure of the software architecture.
\end{abstract}

\section{Introduction}

An ITS provides direct customized instruction or feedback to humans in their sporting processes by means of artificial intelligence techniques, being mainly applied to knowledge representation, managing an instruction strategy as an expert both in the guiding and pedagogical issues in order to diagnose properly the activity status at any time. Traditional way of payment is confined to business hall or agents in artificial service, not only the user payment subject to the conditions of the time and place, and make service providers must spend a lot of manpower and material resources on payment business. In the face of this new business trend, want to develop a new type of service system to provide users with high-quality full-service. Among them, the self-service terminal is a kind of more feasible, it adopts the self-support service mode, not only can improve the efficiency of the service, shorten the user waiting time, and can reduce the work intensity of the front desk staff, make them liberated from repetitive work. Expected goal is based on self-service terminal services system, to meet business function at the same time, must give full consideration to two prominent questions: (1) how to under the condition of network interruption to ensure 24-hour service; (2) how to upgrade in the late kiosks deployment of decentralized reduce maintenance costs. In the existing solutions, basically have C/S architecture and B/S structure to choose from. Among them, the C/S architecture has rich interface, quick response, the advantages of the application server load is light, but the main drawback is a complex and version update application deployment difficulties; B/S architecture has the application easy to deploy, easy to maintain and develop low cost advantage, but the main drawback is the difference in user interaction and rely too much on the network connection. Obviously, these two kinds of architecture cannot meet the requirements of the project, will find a way to both advantages of centralized software architecture design which become the problem that must be solved in the process of development.

ITU Internet of things according to the report, connecting the physical world and virtual reality information with the Internet of things make any time, any place and any items connection possible, not only is the age of the Internet interpersonal connections. Internet of things through the radio frequency identification, sensor and network interconnection technology, realization of signs, perception of physical world objects, monitoring, tracking, control and management, the people and things, people and environment, and implement efficient, intelligent, harmony between objects and things of information interaction, ultimately controlled by the intelligent decision fusion technology to realize the physical world and information world. The Internet of things has become a focus of 
attention of academia and the business and even become one of the development strategies of many countries. Through the IoT infrastructure layer aware of device object information, and then use the cognitive access to huge amounts of data transmission network technology to Internet data center. Internet information service system integrating a lot of data, provide information services for all kinds of Internet applications and receive feedback control.

In this paper, we conduct research on the design and implementation of mobile intelligent service system for city sports venues. Internet of things through a variety of sensing equipment to obtain data has massive, heterogeneous, high dimension, redundancy, time series and space dispersion, etc. At the same time, the open architecture of Internet of things require higher security guarantee mechanism of information service, information service system including security, user privacy protection and the credibility of information service requirements. IoT application objects through all kinds of information service implementation, tracking, monitoring and intelligent control, etc., the design of Internet information service system is the premise to realize the Internet information service. The detailed discussion is conducted in the following sections.

\section{The Designed Platform and System}

The Basic Description of IoT. Physical world objects of large number and variety of forms, and constantly movement change, and distributed in various locations, and easily influenced by the external environment, resulting in the Internet of things through a variety of sensing equipment acquisition with mass of data, heterogeneous, high dimension, redundancy, time series and space dispersion, etc. In addition, in order to save storage space, improve storage efficiency and convenience of data mining, Internet of things must be huge amounts of data for effective pretreatment. The Internet of things from the huge amounts of data according to the knowledge to provide users with a variety of information services, research is suitable for the characteristics of the Internet of mass data of data mining technology is the premise to realize the Internet information service. Internet information service system safe and stable operation without effective security mechanism is crucial. Iot architecture openness, perception of ubiquitous and interaction of frequent sex are security, privacy, and the credibility of the information service put forward higher requirements. To ensure the security of Internet information services, reliable, is the precondition of the Internet of things technology on a large scale application, is necessary for users to accept the Internet information service.

Internet information service system is to provide Internet information service platform, and realize the carrier of Internet application. Internet information service system is for huge amounts of data, the top bottom for large user base, and services in a variety of forms, so the design of Internet information service system is different from the traditional enterprise information system design. In Internet of mass data processing, according to the characteristics of Internet of vast amounts of data need to be further discussed simple, efficient and reliable mass data storage technology. For iot huge amounts of data preprocessing in as much as possible to remove redundancy should be fully considered at the same time and does not affect the integrity and authenticity. In distributed heterogeneous integration of IoT in huge amounts of data mining, data code to further research and extracting technology, statute or extracted from huge amounts of data, and excavated the characteristics of knowledge required in an efficient and comprehensive data sets. For should not be simplified or difficult to simplify the complex of huge amounts of data mining, to focus on problem of efficiency and considering the complexity of the algorithm. On Internet information service system of security, can, on the basis of traditional information security technology research more suited to the characteristics of the Internet information service security mechanism.

The Architecture of the Client Port. "Smart client" as a new kind of software architecture, is put forward by Microsoft developers in 2004 day. Through design, it will let C/S and B/S structure combined with the advantages of the formation of a new model of software development. Generally speaking, smart client is an easy to deploy and manage application architecture, through the use of local resources as a whole and smart way to connect to the distributed data resources, provide strong 
adaptability, rapid response and rich content of interactive experience. Smart client is generally divided by the Web service client application and server in two parts. Among them, the client is responsible for providing users with interactive features, generally by the presentation layer, business layer and data layer; Responsible for dealing with client service request on the server, generally by the Web of interface layer, business layer and data layer. Both mainly by the client service layer and server Web interface layer calls communication feedback. As a new software solution, smart client has many improvements and innovations on architecture design. It make full use of local resources and on the basis of Web services, put forward the connection and occasionally work smart update strategy, and provides the corresponding software package for the practical application, for secondary development gave a powerful technical support. At the same time, the client and server side there are two important interaction module, namely to work offline module and smart update module. Work offline module is responsible for the client online and offline and server-side function flow switch, smart update module is responsible for the client and server application version of synchronization. The user interface is shown in the figure 1.
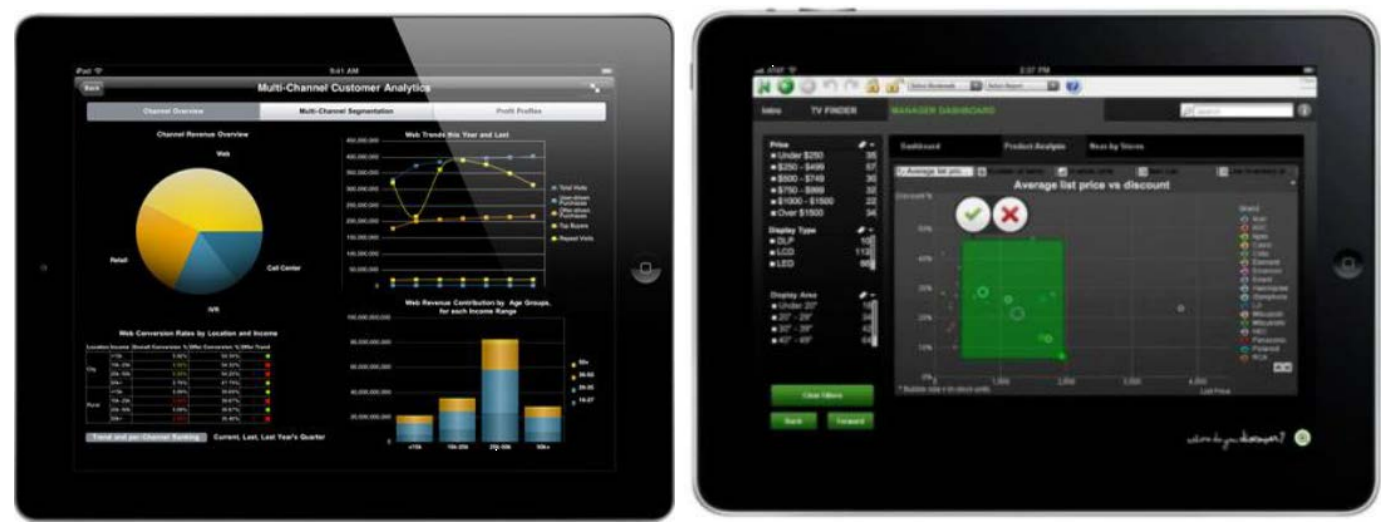

Fig.1The User Interface of the Proposed Client

The Design Concepts and Principles. Conventional information systems (CIS) including decision-making support system or analytics system have fixed service flow and support only static services. Whereas the proposed system which employs user-adaptive service flow and can dynamically change service flow reflecting user intention obtained from users' behavior patterns as well. Work offline as smart client application an important characteristic different from other types of applications, is through the establishment of occasionally connected strategy implementation. In the past, due to the component must be registered and install the application documents, the client application deployment and upgrade become very difficult. Now, the.net framework by supporting simply copy files to the appropriate directory to complete the installation, the large-scale deployment of client application. Ideally, the administrators on a central server to deploy the updated files only once and after the client through the version detection mechanism automatically detects whether to need to download a replacement, and the new version of the program or component does not need to register can be used directly. This is the main design ideas, smart update strategy, of course, in the actual application will involve the issue of safety and efficiency. In smart client application, there are two ways to realize the occasional connectivity, namely a data-centric method and service-oriented. (1) Data-centric approach is installed in the local database management system, and use the built-in functionality to the local data changes back to the server, processing synchronization process, and to detect and solve any data conflict. (2) Service oriented approach is to store data information in the message, when the client is offline, arrange these messages to the queue when to establish a connection queue of messages will, in turn, is sent to the server for processing. (3) In the past, due to the component must be registered and install the application documents, the client application deployment and upgrade become very difficult. Now, the.net framework by supporting simply copy files to the appropriate directory to complete the installation, the large-scale deployment of client application. Ideally, the administrators on a central server to deploy the updated files only once and 
after the client through the version detection mechanism automatically detects whether to need to download a replacement, and the new version of the program or component does not need to register can be used directly. This is the main design ideas, smart update strategy, of course, in the actual application will involve the issue of safety. The intending interface and flowchart core technique is shown in the figure 2 .

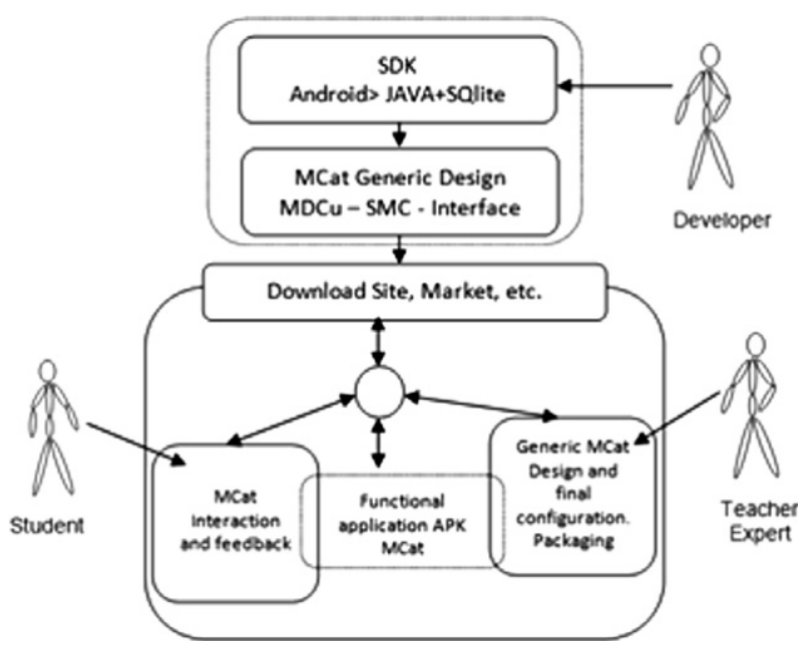

Fig.2 The Intending Interface and Flowchart Core Technique

\section{Conclusion and Summary}

Traditional way of payment is confined to business hall or agents in artificial service, not only the user payment subject to the conditions of the time and place, and make service providers must spend a lot of manpower and material resources on payment business. In the face of this new business trend, want to develop a new type of service system to provide users with high-quality full-service. Among them, the self-service terminal is a kind of more feasible, it adopts the self-support service mode, not only can improve the efficiency of the service, shorten the user waiting time, and can reduce the work intensity of the front desk staff, make them liberated from repetitive work. In this paper, we conduct research on the design and implementation of mobile intelligent service system for city sports venues. Internet of things through a variety of sensing equipment to obtain data has massive, heterogeneous, high dimension, redundancy, time series and space dispersion, etc. The proposed system help to modify the current situation of sport service and in the future, we plan to combine more related IoT technique to enhance the robustness of the current system.

\section{Acknowledgements}

Social science fund project in hebei province(HB12TTY014).

\section{References}

[1] Xian-li L, Shu-yuan Z, Center I. A Study on Intelligent Customer Service System Based on Mobile Platform[J]. Journal of Inner Mongolia University(Natural Science Edition), 2014.

[2] Yuansheng L, Liangyi C, Xiangyang X. Customer Loyalty Management System Development for Auto 4S Dealers Based on Intelligent Mobile Terminal[J]. Customer Loyalty Management System Development for Auto 4S Dealers Based on Intelligent Mobile Terminal - ResearchGate, 2013, 33(4):767-771.

[3] Bayliss P R, Cohen R A, Collins G O, et al. Intelligent global services bus and system for mobile applications: WO, WO2013059661 A1[P]. 2012. 
[4] PD K. Cloud based intelligent system for delivering health care as a service.[J]. Computer methods and programs in biomedicine, 2014, 113(1):346-359.

[5] Balakuntala S, Venkatesh S. An Intelligent System to Detect, Avoid and Maintain Potholes: A Graph Theoretic Approach[J]. Mobile Computing and Ubiquitous Networking (ICMU), 2014 Seventh International Conference on, 2013:80. 